\title{
Multimedia Communication Systems: Upper Layers in the OSI Reference Model
}

\author{
Mirjana Zafirovic-Vukotic and Ignas G. Niemegeers, Member, IEEE
}

\begin{abstract}
Future multimedia systems will rely on broadband networks and subnetworks which will be able to provide the required type of communication services and will meet the stringent quality of service (QOS) demands. However, it is likely that the introduction of multimedia communication will have to be based on networks providing simple services that are not directly meeting the multimedia requirements. This paper starts from this assumption and focuses on the end systems. Within the framework of the OSI Reference Model, it examines the functionality and structuring of the higher protocol layers required to support multimedia communication. Furthermore, it discusses some important implementation and performance issues.
\end{abstract}

\section{INTRODUCTION}

$\mathrm{F}$ UTURE multimedia systems will rely on broadband networks, e.g., the B-ISDN [2], [3], and subnetworks which will be able to provide the required type of communication services and meet the stringent quality of service (QOS) demands. However, many subnetworks that are existing or under design will not be sophisticated enough to provide the services with the right characteristics. This will require the use of sophisticated multimedia end systems which are able to compensate for the deficiencies of the networks. In particular, the structuring and functionality of the higher layer protocols will be affected. This shall impact the introduction strategy of multimedia applications. It is likely that, initially, use will be made of a simple transport layer service, which in turn will be implemented on top of various network services.

This paper focuses on the upper layers of the OSI Reference Model [14]. It examines the structuring, services, and major protocol functions that are required in the upper layers in order to support end-to-end multimedia communication, assuming a simple transport service. It is also assumed that variable bit rate (VBR) coding techniques will be used in future multimedia systems. Mutual stream synchronization, integral management, and multiparty connections appear to be crucial issues in multimedia communication and are addressed in this paper. Important implementation issues and performance issues are discussed.

Manuscript received January 1992; revised June 1992

M. Zafirovic-Vukotic is with the M. Pupin Institute, 11000 Beograd, Yugoslavia.

I. G. Niemegeers is with the University of Twente, 7500 AE Enschede, Holland.

IEEE Log Number 9204271
The paper starts with a survey of related research in Section II. Ideas about the required services, protocol functions, and structuring of the upper OSI layers are proposed in Section III. Implementation and performance issued are discussed in Sections IV and $\mathrm{V}$ respectively. Conclusions and future work are outlined in Section VI.

\section{State of the ART}

Let us consider multimedia end systems consisting of an application process and an open-ended multimedia communication system, which supports the simultaneous use, manipulation, and transfer of diverse types of information within a single communication instance with other such systems. Strongly different types of information are referred to as media, and instances of information exchange of a particular type are called streams. Examples of streams are compressed video, voice, interactive data, and graphics. An implementation of an end system will be called a terminal.

Research in broadband end systems and the terminal architecture on which they are based has led to a number of reference models. The BERKOM implementation model [20] is one of them. It integrates the OSI Presentation and Application layer and proposes the use of a common data structure for multimedia applications. In [9], a vertical structuring for the upper layer protocols is proposed with the intent of exploiting parallel processing. A protocol design for multimedia communication has been proposed in [24]. In [10], a multimedia multiuser service for B-ISDN has been described which abstracts from possible implementations. In particular, multiuser call establishment has been explored. Multimedia terminals are already available on the market, however with a rather modest degree of media integration, e.g., systems integrating video and digital voice or video and graphics. An example of an experimental prototype has been reported in [12].

Narrowband networks such as the ones based on X.25 and the ISDN are only to a limited extent usable for multimedia communication because some media require a high throughput. Broadband communication networks, on the other hand, which will be able to provide throughputs of tens of $\mathrm{Mb} / \mathrm{s}$ per connection are fully enabling multimedia communication. Research and standardization in B-ISDN show an inclination towards placing some functions into the network such as code conversion, informa- 
tion compression, and multimedia stream synchronization, even though these functions are generally not considered to fit into the lower three OSI layers [14]. However, in this way the terminal equipment can be kept simpler at the expense of the network nodes which become significantly more complex. Counter-arguments for this approach can be found in [25], where simplicity in the first designs of the B-ISDN is advocated. Note that the present standards for HSLAN's and MAN's define integrated services which are not particularly suited for multimedia and which can be characterized as "simple" in the context of multimedia communication.

According to the bit rate of the service, two classes of transfer (bearer) services can be distinguished: VBR transfer services and constant bit rate (CBR) transfer services. Characteristics of a VBR transfer service are the variable data transfer delay and irregular servicing of the queues of packets waiting for transmission. The service rate may possibly be guaranteed but the interservice time is variable. This type of service typically supports VBR traffic which is generated by applications such as interactive data, file transfer, electronic mail, document exchange and graphical image transfer, and also by applications which require a constant end user-to-end user delay like (VBR coded) video, voice, and HDTV. A constant bit rate (CBR) transfer service is characterized by a constant data transfer delay and a periodic availability of service quanta at the packet queues. It typically supports CBR traffic like voice, video, and HDTV when they appear as CBR sources. CBR transfer services are generally related to circuit-switched implementations which make use of fixed bandwidth reservation. VBR subnetwork or lower layer transfer services are related to implementations which are based on packet switching, which enables statistical resource sharing. Note that this classification of transfer services is included in the ATM AAL classification of services given in [3]. It is not based on timing requirements.

Broadband communication subnetworks can be classified according to the classes of the OSI Subnetwork Specific 3A or the Datalink Layer (DLL) services into two categories: those providing a VBR transfer service and those providing both CBR and VBR transfer services. Examples of the former are frame relaying networks [21], 2 $\mathrm{Mb} / \mathrm{s}$ X.25 upgrades [23], CFR [11], Orwell [7], and Metrobridge [27]. Examples of the latter are FDDI-II [15], IEEE 802.6 [13], and B-ISDN [2], [3]. Transport layer services and protocols for broadband networks are an active research area (see, e.g., [5], [16], and [17]). Transport protocols have end-to-end significance. They make use of diverse network services, which in general are internetting services based on the $3 \mathrm{~A}$ or DLL services. Those services are so far not particularly suited for multimedia support with the possible exception of the services that will be provided by the B-ISDN. Therefore, one can expect that in the first stages of the introduction of multimedia communication, transport protocols will be used that are based on such network services, resulting in simple transport services which are not particularly suited for multimedia support.

In this paper, we assume the standardized services, protocols, and functional blocks of the higher OSI layers and we propose novel additions to them to support multimedia communications. In line with the research effort, e.g., in the European COST Project 229 (Applications of Digital Signal Processing to Communications), and the present developments in CCITT (e.g., layered coding has been suggested in CCITT Recommendation I.211 for B-ISDN [4]), a VBR coding technique for video is assumed. For a constant quality picture, it provides a lower average stream intensity but also a variable stream intensity as compared to CBR coding. This paper further assumes a VBR subnetwork service and a corresponding "simple" VBR transport service. The terminal equipment may be quite complex. In the context of multimedia communication, mutual stream synchronization, integral management, and multiparty connections appear to be particularly interesting issues for the design of the upper OSI layers.

\section{Protocol Functions, Structuring, and SERVICES}

Let us now present our views on the issues related to the protocol functions, structuring, and services in the upper OSI layers for multimedia communication. This section is organized into subsections dealing with the application layer, presentation layer, session layer, multiplexing and concatenation, and management.

\section{A. Application Layer}

Application layer multimedia communication is inherently connection-oriented. We believe that multimedia communication can best be realized by a single application layer connection. This simplifies multimedia call establishment, release and control, authentication and authorization, and management. An illustration of the proposed architectural concept is shown in Fig. 1.

Usually in multimedia multiparty applications, a master station is designated. The application process at the master station is the one who give turns in multiparty communication. In addition, it is possible that each station sends information to the master station where the application process selects information, maybe processes or merges it, and then sends identical data to each participant. An alternative is that the participant who has the turn in a multimedia application sends data to all participants directly. In any case, a multimedia multiparty connection at the application layer can be considered a centralized multiendpoint connection. Furthermore, a central communications control and management system can be efficiently realized at the master station, e.g., the addition of a new participant or the recovery of a failed one in a multiparty connection asks for refined resynchronization functions which can more easily be handled centrally by a master station than in a distributed way. In the lower 


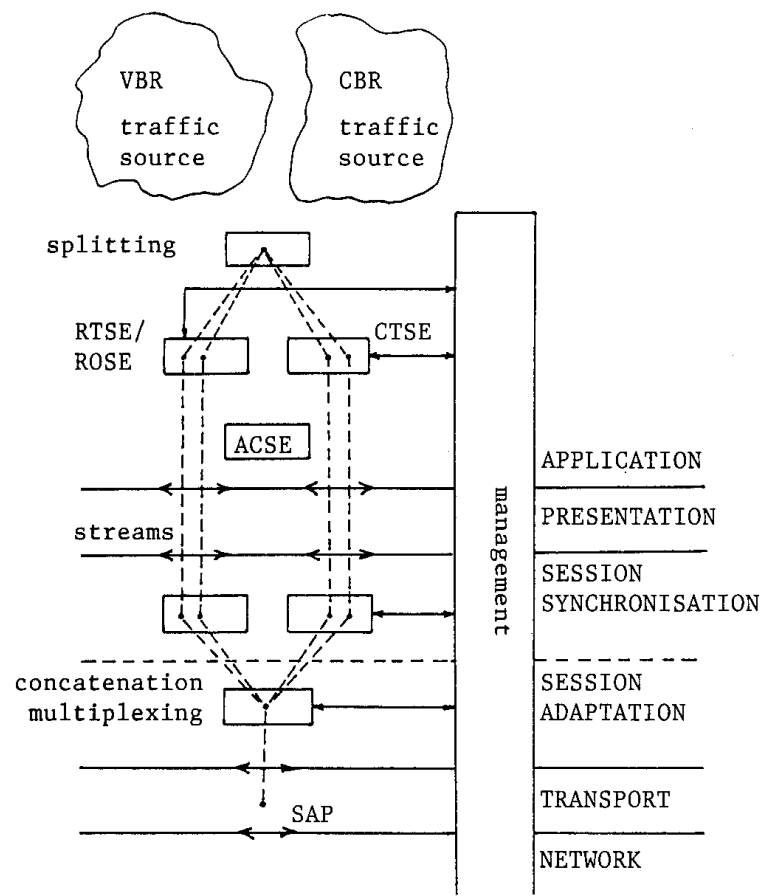

Fig. 1. Illustration of the upper OSI layer structure with major elements, functions, and interfaces.

OSI layers, use can be made of multipoint and multicast communication services, e.g., in B-ISDN and HSLAN's,

The streams require diverse lower application layer services. Already in the lower part of the application layer, connections are to be implemented using protocol functions between the peer entities as has been done in the standardized application blocks. This implies that, in the lower part of the application layer, separate connections have to be used for the streams. Thus, a splitting function is to be used within the application layer.

An Association Control Service Element (ACSE) is used within the application layer to provide for connection establishment and release of each data stream. Reliable Transfer Service Elements (RTSE), Remote Operations Service Elements (ROSE), or similar elements (which, as a rule, use ACSE service) are used for VBR data transfer. For CBR end users, e.g., audiovisual users before coding is implemented, a similar element should be specified. Let us name it CBR Transfer Service Element (CTSE). It uses ACSE services and provides itself the corresponding services, controls the stream, transparently transfers information between its service user and the presentation layer entity, and provides other functions which are to be investigated further e.g., functions related to data presentation under windows.

Therefore, the application layer should provide the following major functions:

- a single (unique) connection for a multimedia call which can be a multipoint connection

- a splitting function, splitting a single multimedia call into a number of stream connections
- connections for each data stream in the lower part of the application layer, and

- multimedia connection integrity control, i.e., control of service elements and their function in a multimedia connection

\section{B. Presentation Layer}

The presentation layer service is to be provided separately to each stream. A single association is mapped onto a single presentation layer connection. Negotiation of the communication contexts is possible and significantly different data presentation techniques, e.g., coding techniques, can be available for diverse streams. The presentation layer, thus, provides specific presentation functions for each data stream separately. The VBR codec functionality should be part of the presentation entity.

\section{Session Layer}

The session service is to be provided to each stream separately. For example, session connection synchronization is available for each stream separately, e.g., activity dialog, major and minor token features, or activity token service which enables half-duplex communication. A presentation connection is mapped on a session connection. On the other hand, it is required that mutual synchronization of the streams is performed in the session layer since the underlying transport service is assumed to be simple and not to offer any such service.

The session layer is organized into the following two subsessions: an upper one (synchronization) and a lower one (adaptation). Both sublayers provide for a connection-oriented service. The synchronization sublayer performs functions which are common for the session layer according to the OSI Reference Model. For example, the synchronization sublayer performs connection establishment and release, dialog management, and synchronization and resynchronization of each stream in a multimedia conversation. The adaptation sublayer performs multiplexing of data streams into a small number of connections, e.g., two transport multimedia connections; furthermore, it handles concatenation providing constant data transfer delay, treatment of special PDU's, and management of particular data streams. The concatenation function is used for connections for which the multiplexing function is used as well. Concatenation is a function in the session layer [14]. An option in the OSI Reference Model which was left for possible future use allows multiplexing within the session layer [14]. This option is to be used in multimedia communication.

The session layer should provide the following major functions and service characteristics:

- connection synchronization for each stream like dialog management

- flow control by means of back pressure to the streams separately and to the multiplexed components of the integral multimedia communication

- mutual synchronization of the streams 
- stream multiplexing

- concatenation

- constant data transfer service duration, and

- management of each data stream and the multimedia communication

\section{The Multiplexing and Concatenation Issue}

In general, the traffic streams do not need to be multiplexed (see [8] for a study of multiplexing as a protocol function). However, if multiplexing and concatenation are not used as a session layer function, large fluctuations are possible in the transfer delay of the information which belongs to different connections and, therefore, to different streams.

On the other hand, subnetwork bandwidth allocation and congestion control do not necessarily require that a distinction be made between different VBR information types (including VBR video) and between the corresponding streams within a single subnetwork such as the B-ISDN or a HSLAN. The bandwidth allocation can be handled as follows. Since different streams are handled by different application layer, presentation layer and synchronization sublayer entities, large fluctuations in the traffic load, e.g., the start of a file transfer, can be signaled during the multimedia conversation to the adaptation sublayer or transport layer connection(s) which, in turn, can signal it to the lower layer connections(s). This is in line with the virtual circuit (VC) and virtual path (VP) concepts in B-ISDN (see, e.g., [1]) and the HSLAN VBR services. The subnetwork congestion control can signal the congestion state or impending congestion, and end-to-end flow control can be used to resolve the congestion (see, e.g., [6] and [22]). End-to-end flow control is performed by the transport protocol and possibly by the lower layer protocols. Thus, the control is done over the multiplexed components of the integral multimedia connection. Flow control can also be exerted by means of back pressure in the multiplexed components of the integral multimedia connection and in each data stream in the session layer. Such flow control can help the network congestion control function. It also enables the use of different stream intensity control techniques used by adaptive coding techniques. The effectiveness of such a congestion control scheme depends on network monitoring and response time. It is expected that, due to the technological progress, this will not be a problem. Note that only two traffic classes are distinguished in ATM [19]. Studies of diverse subnetworks indicate that this number is sufficient for congestion control to ensure the required QOS (see, e.g., [26]).

The purpose of concatenation is to ensure a constant packet delay, i.e., a constant adaptation sublayer service duration. The adaptation sublayer protocol also supports particular PDU's which have their own PDU format and possibly their own connection endpoint identifier. These PDU's can be of use in exception handling and other procedures.

\section{E. Management}

Multimedia end user connection establishment, release, and control relies on management functions. Essentially, it is important that the management entity of the application and other concerned layers have full information on the requirements and status of the multimedia connection. The application layer management entity and systems management need full coordination. The application layer management entity has a natural role to trigger functions of the other layers. It also provides an interface to the application process and its management. In particular, multimedia application management should perform, among others [14], the following functions:

- integrity and commitment control

- checkpointing and recovery.

\section{ImPlementation Elements and Complexity}

VBR coding and decoding in a multimedia terminal is considered to be part of the presentation layer functionality. Therefore, the interface between the presentation layer and the application layer which also provides for the interaction with the application process, and the interface between the presentation layer and the session layer which also provides for interaction with the underlying subnetwork, should include all the interactions required by the VBR codec except those provided by the management entities.

Note that this functionality may also be required in broadband network nodes in order to enable code conversion [18].

Management elements should also be integrated into a multimedia terminal in order to provide particular information required for coding and decoding. An example is the maximum data rate which is allowed. This, in turn, is related to flow control. The management elements of the application layer, session layer, multimedia application, and systems management play an essential role in the coordination of flow control of the data streams and the multimedia connection support. The complexity of the group of management elements is rather large. Note that QOS requirements of specific streams and the status of the connections for each stream should be available to the management elements.

The inherent parallelism which is apparent in the processing and connection management of the upper layer protocols for multimedia communication suggests the possibility of an implementation on a processor with a parallel architecture (see, e.g., [9]). This may be a way to achieve the high throughput requirements and satisfy the real-time constraints.

Given that there is a single VBR transport service, one can guarantee a constant duration of the session data transfer by using an element which polls the streams at regular time intervals (see Fig. 2). At the source, this element constructs composite data packets (concatenation) and, at the destination, it unpacks these packets (separation). One or more of such elements can be used, depend- 


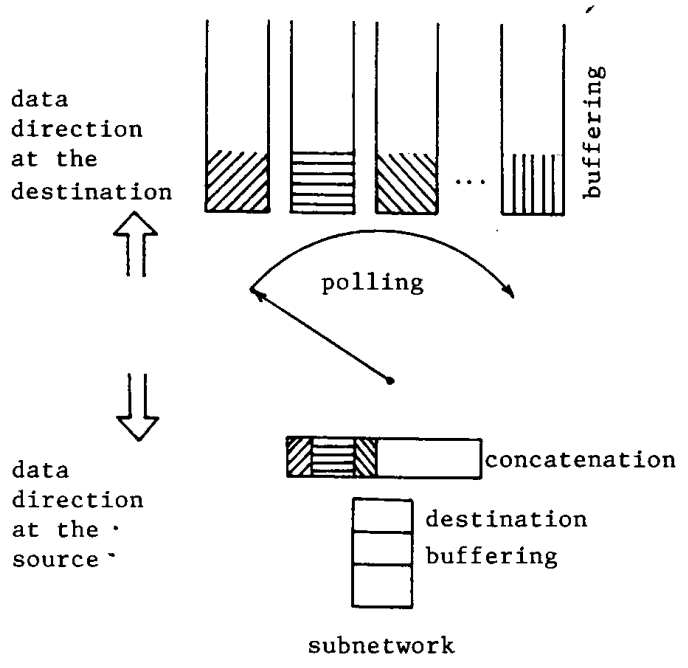

Fig. 2. Illustration of packet assembly.

ing on the number of underlying connections to which the multiplexing relates. On the one hand, increasing the number of such elements makes the implementation more complex but on the other hand the polling speed becomes less critical and parallel processing can be used. To allow a variable transport layer service delay and a constant synchronization service delay, the polling is additionally delayed at the destination. Both at the source and destination, streams may have to be additionally delayed to cope with the variable duration of presentation layer processing. The largest presentation layer delay of the streams which are nowadays used is the compressed video coding and decoding delay. Therefore, the total data delay in a multimedia connection equals the compressed video delay. Additional storage is needed to buffer data in order to provide a constant delay between the two end users.

\section{Performance Considerations}

Let us now consider the packet delay in the data transfer phase, which is due to the protocol processing in the upper layers for point-to-point communication.

In the lower session sublayer, connection multiplexing and packet concatenation are performed. The delay due to this is determined by the cycle time of a stream polling cycle. The optimal cycle time in multimedia communication is related to the following two parameters: the packet processing time and the transmission rate which in turn influences the packet length. In the literature, this issue is discussed for fixed length packets (cells). Note that the polling element is placed in the session layer entity and that the session PDU (packet) has a variable length. Similarly to the packet concatenation, the packet separation lasts for a cycle which has the same length as the one at the source.

Let $z$ denote the cycle duration. To allow a variable transport layer service delay and a constant synchronization service delay, the polling is additionally delayed at the destination. Let us denote by $k$ the mean number of buffered and delayed packets at any instant in time due to this. It depends on the transport layer service delay variance. The total mean delay due to the buffering is, therefore, not larger than $(2+k) z$.

The largest presentation layer delay is expected to be due to the coding and decoding of compressed video. Let us denote it by $y$. To synchronize all streams, it may be necessary to delay some of them. Let $I_{i}$ indicate whether buffering is used or not for stream $i$ in order to provide such extra delay. Let $I_{i}$ take the value 1 if such buffering is used and value 0 otherwise. The total mean packet delay due to buffering is, then, $2 y I_{i}$. Protocol processing also contributes to the delay in the upper OSI layers, e.g., in the application layer. Let us approximate this delay by $2 x$. The three previously determined components make us conclude that the total expected packet delay due to upper layer protocol processing $t_{i}$ amounts to the following:

$$
t_{i}=(2+k) z+2 y I_{i}+2 x \text {. }
$$

The first two elements in the sum are related to the use of VBR coding techniques. The factor $k$ is related to the stochastic properties of the transport and subnetwork services. An increase of the cycle duration $z$ does not imply an increase of $k$. On the contrary, it can be expected that the value of $k$ decreases. So, the factor $2 z$ linearly depends on $z$, and the factor $k z$ includes two parameters which seem to be inversely proportional. This implies that the use of a relatively long cycle time $z$ could provide a satisfactory performance $t_{i}$, which is to be investigated further.

\section{Vi. Conclusions and Future Work}

The protocol functions, elements, and services which have been proposed in this paper for the support of multimedia communication constitute one of the first attempts at analyzing the structure of the upper OSI layers in light of multimedia communication.

A simple transport layer service was assumed, which in turn may use different subnetwork services. This is thought to be a realistic approach for the first stage in the introduction strategy of multimedia applications. Starting from the existing standards, a proposal was presented which, to a large extent, conforms to the OSI model and uses the multiplexing option in the session layer. Concatenation is performed in the session layer and splitting is done in the application layer. A number of issues are raised regarding implementation complexity, in particular the complexity of the upper layer management, processing parallelism, the realization of constant delay, and stream synchronization. A tradeoff is discussed which exists between the gain obtained by VBR coding techniques and the efficient use of subnetwork capacity and resources involved in the upper layer protocol handling.

The paper presents a basis for further investigations of the related protocols and subnetwork workload modeling. In particular, performance tradeoffs will have to be evaluated. 


\section{REFERENCES}

[1] J. D. Burgin, "B-ISDN resource management: The role of virtual paths," IEEE Commun. Mag., vol. 29, no. 9, pp. 44-49, Sept. 1991.

[2] CCITT, "B-ISDN ATM adaptation layer (AAL) specification," Draft Recom. 1.363, Geneva, Switzerland, June 1990.

[3] CCITT, "B-ISDN ATM adaptation layer (AAL) functional description," Draft Recom. I.362, Geneva, Switzerland, June 1990

[4] CCITT, "B-ISDN service aspects," Draft Recom. I.211, Geneva, Switzerland, June 1990.

[5] W. A. Doeringer, D. Dykeman, M. Kaiserswerth, B. W. Meister, H. Rudin, and R. Williamson, "A survey of lightweight transport protocols for high-speed networks," IEEE Trans. Commun, vol. 38, no. 11, pp. 2025-2039, Nov. 1990.

[6] A. E. Eckberg, B. T. Doshi, and R. Zoccolillo, "Controlling congestion in B-ISDN/ATM: Issues and strategies," IEEE Commun. Mag., vol. 29 , no. 9, pp. 64-74, Sept. 1991

[7] R. M. Falconer and L. Adams, "Orwell: A protocol for an integrated services local network," British Telecom. Tech. J., vol. 3, no. 2, pp. 85-91, Apr. 1985

[8] D. C. Feldmeier, "Multiplexing issues in communication system design," in Proc. ACM SIGCOMM'90, Sept. 1990, vol. 20, no. 4, pp. 209-219.

[9] Z. Haas, "A protocol structure for high-speed communication over broadband ISDN,"' IEEE Network Mag., pp. 64-70, Jan. 1991

[10] G. J. Heijenk, M. A. Jordense, R. A. Sturrus, and I. G. Niemegeers, "A multimedia multiuser service for the B-ISDN," in Proc Memorandum Informatica 91-56, University of Twente, Enschede, The Netherlands, June 1991.

[11] A. Hopper and R. M. Needham, "The Cambridge fast ring networking system," IEEE Trans. Comput., vol. 37, no. 10, pp. 1214-1223, Oct. 1988.

[12] A. Hopper, "Pandora-An experimental system for multimedia applications," Operat. Sys. Rev., vol. 24, no. 2, pp. 19-34, Apr. 1990

[13] IEEE, "Distributed queue dual bus (DODB) subnetwork of a metropolitan area network (MAN),"' IEEE 802.6 Standard, Dec. 1990.

[14] ISO 7498, "Information processing systems-Open systems interconnection-Basic reference model,"' 1984.

[15] ISO DIS 9314-5, "FDDI hybrid ring control," ISO Draft Int. Standard, 1991.

[16] N. Jain, M. Schwartz, and T. Bashkow, "Transport protocol processing at GBPS rates,' in Proc. ACM SIGCOMM'90, Sept. 1990 vol, 20, no, 4, pp. 188-199.

[17] T. F. La Porta and M. Schwartz, "Architectures, features and implementation of high-speed transport protocols," IEEE Network Mag., pp. 14-22, May 1991

[18] S. E. Minzer and D. R. Spears, "New directions in signaling for broadband ISDN," IEEE Commun. Mag., vol. 27, no. 2, pp. 6-14, Feb. 1989

[19] T. Okada, H. Ohnishi, and N. Morita, "Traffic control in asynchronous transfer mode," IEEE Commun. Mag., vol. 29, no. 9, pp. 5863, Sept. 1991

[20] R. Popescu-Zeletin, "From broadband ISDN to multimedia computer networks," Comput. Networks and ISDN Sys., vol. 18, pp. 47-54, 1989/1990.
[21] M. Rahnema, "Frame relaying and the fast packet switching concepts and issues," 'IEEE Network Mag., pp. 18-23, July 1991

[22] J. W. Roberts, "Variable bit rate traffic control in B-ISDN," IEEE Commun. Mag., vol. 29, no. 9, pp. 50-57, Sept. 1991

[23] B. Sales, "A profile for wide area X.25 operating at $2 \mathrm{Mb} / \mathrm{s}$," Comput. Networks and ISDN Sys., vol. 21, no. 4, pp. 307-314, June 1991.

[24] H. Shimizu, M. Mera, and H. Tani, "Packet communication protocol for image services on a high-speed multimedia LAN," IEEE J. Select. Areas Commun., vol. 7, no. 5, pp. 782-788, June 1989.

[25] S. M. Walters, "A new direction for broadband ISDN," IEEE Commun. Mag., vol. 29, no. 9, pp. 39-43, Sept. 1991

[26] M. Zafirovic-Vukotic, I. Niemegeers, and D. Valk, "Performance analysis of slotted ring protocols in HSLAN's," IEEE J. Select. Areas Commun., vol. 6, no. 6, pp. 1011-1024, July 1988

[27] K. Zielinski, M. Chopping, D. Milway, A. Hopper, and B. Robertson, "The Metrobridge, a backbone distributed switch," Comput. Commun. Rev., vol. 21, no. 3, pp. 45-60. July 1991.

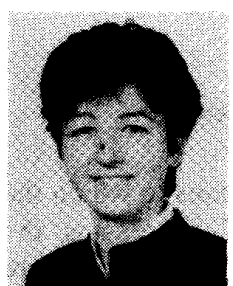

Mirjana Zafirovic-Vukotic received the B.S. degree in mathematics with a minor in computer science, and the M.S. degree in operations research from the University of Belgrade, Belgrade, Yugoslavia, in 1981 and 1984, respectively, and the $\mathrm{Ph} . \mathrm{D}$. degree in engineering from the University of Twente, Enschede, Holland in 1988

She is currently a Researcher at the M. Pupin Institute at the University of Belgrade, Belgrade, Yugoslavia, and an Assistant Professor at the Electrical Engineering Faculty of the same university. Her current interests are in communication systems and performance modeling and analysis.

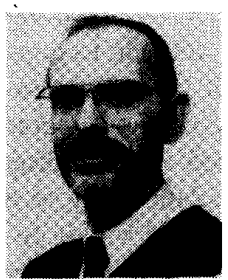

Ignas G. Niemegeers ( $\left.S^{\prime} 70-M^{\prime} 78\right)$ was born in Gent, Belgium, in 1947. He received the degree in electrical engineering from the Rijksuniversiteit Gent in 1970, and the MSc. E. degree in computer engineering and the Ph.D. degree from Purdue University, West Lafayette, IN, in 1972 and 1978, respectively.

From 1978 to 1981 , he was employed by the Research Department of Bell Telephone Manufacturing Company, Antwerp, Belgium, where he was involved in the design of packet switching systems. In 1981, he joined the Department of Electrical Engineering, University of Twente, Enschede, The Netherlands, as an Associate Professor. He was responsible for teaching and research in computer networks. In 1986, he got a Full Professorship in the Department of Computer Science at the University of Twente. His areas of interest are communication systems and performance analysis. He is active in research on integrated networking, high-speed networking, B-ISDN, ATM switching, and performance analysis and performability. 